\title{
Brazilian Food Guide attacked: now, overwhelming support for the Guide in Brazil and worldwide
}

By Carlos Monteiro and Patrícia Jaime*

*Coordinator and deputy scientific coordinator of Nupens/USP and full professors at the Department of Nutrition of the School of Public Health of USP

\section{Abstract}

The present commentary summarizes the attacks to the Dietary Guidelines for the Brazilian Population from ultra-processed food manufacturers and the Brazilian Ministry of Agriculture, Livestock and Supply (MAPA). After MAPA issued a technical note calling on the Ministry of Health urgently to review the Guide by cutting out its recommendation to avoid consumption of ultra-processed food products, without citing scientific evidence, a wide group of scholars confirmed that the note had no valid foundation.

Key words: Ultra-processed food, Food processing, Food classification, NOVA

\section{Background}

Defining healthy diets is relatively simple. But confusion and controversies in the media can suggest otherwise. A lot of commotion is caused by sources motivated by profit and not by personal and public health, as this commentary will show.

Making the scientific knowledge on healthy diets accessible to everybody is one of the purposes of the world-leading Dietary Guidelines for the Brazilian Population (the Food Guide) published by the Ministry of Health (Ministério da Saúde, Brasil, 2014) The Guide was produced with the technical support of the Center for Epidemiological Research in Nutrition and Health of the University of São Paulo (Nupens/USP), as directed by the Brazilian Ministry of Health, in consultation with the Pan American Health Organization of the World Health Organization. It is the product of a thorough national consultation. In draft it was circulated for review and comment by nutrition professionals from all Brazilian states, by non-governmental organizations, and by the 
food industry. It has been acknowledged in reports from the United Nations Food and Agriculture Organization and by the Pan American Health Organization.

The Guide is for absolutely everybody. Its central message can be summed up as "unpack less, and peel and cook more.” The concept of peeling and cooking more, refers to unprocessed foods, such as varieties of fruits and vegetables, and all sorts of minimally processed foods, such as the Brazilian staples beans and rice and modest amounts of animal foods. The Guide states that eating such foods in the form of dishes and meals helps ensure consuming in a balanced way the nutrients and other bioactive compounds that we need to maintain our health and well-being. It also stimulates and supports businesses, farmers and workers who make, produce, distribute and market these foods. Further, it sustains biodiversity, protects natural resources and the environment, and affirms an important part of national and regional culture.

The concept of unpacking is related to consumption of those food products made by manufacturers that are ultra-processed. The Guide states that the more that ultraprocessed foods are eaten or drunk, the greater the risk of consuming excessive sugar and unhealthy fats, and insufficient protein, dietary fibre, vitamins, minerals and other bioactive compounds. Ultra-processed foods also contain many additives which, while used legally, have unknown or uncertain effects on health. Their consumption discourages family farming, decreases biodiversity, threatens natural resources, increases solid waste, and replaces genuine food crops.

The food industry as a whole is of course essential for all societies and civilisations. Indeed, the Guide emphasizes that only some industrially manufactured food is ultraprocessed. Foods can be minimally processed (such as rice and beans), can be processed culinary ingredients (such as oils, olive oils and butter) and can be processed foods (such as freshly-made breads and cheeses).

The Guide states that unprocessed or minimally processed foods are the basis of healthy eating. Processed culinary ingredients, in small quantities, turn those foods into delicious and nourishing dishes and meals. Processed foods, also in small quantities, can make meals more delicious while retaining their nutritive value.

The only foods that the Guide recommends avoiding, are those that are ultra-processed. These are not exactly foods, but formulations of various ingredients, many of them manufactured for exclusive industrial use, very often containing little or no whole food. Examples are cola and soft and sweetened drinks; candies and confectionery; packaged fatty, salty or sugary snacks, cakes and biscuits; “instant” noodles and soups; products made from reconstituted meat; and pre-prepared chilled or frozen pizzas, pastas and other dishes.

The Guide includes a practical way to recognise when foods are ultra-processed. This is by consulting the list of ingredients that, by law, must appear on all packaged foods 
with more than one ingredient: “A high number of ingredients (often five or more) and, mainly, the presence of ingredients whose names are unfamiliar and are not used in culinary preparations (such as hydrogenated vegetable fat, inter-esterified oils, fructose syrup, protein isolates, bulking agents, thickeners, emulsifiers, colorants, flavour enhancers and several other types of additives) indicate that a product belongs to the category of ultra-processed foods.”

\section{Attacks from ultra-processed food manufacturers}

The manufacturers of ultra-processed food in Brazil are now trying to discredit the Guide. In mid-September this year, a "Technical Note" prepared by two officials in the Brazilian Ministry of Agriculture, Livestock and Supply (MAPA) leaked to the media. The Note called on the Ministry of Health urgently to review the Guide, claiming that it was "one of the worst on the planet," and in particular to cut out its recommendation to avoid consumption of ultra-processed food products, without citing any relevant supporting evidence. The Note ignored the vast number of reviews that welcome the Guide and the voluminous world-wide scientific literature linking ultra-processed foods with many chronic diseases.

It is known that in mid-year Brazilian Food Industry Association (ABIA) staff met with the Agriculture Minister to press for changes in the Guide. A few days after the leak, ABIA issued a document supporting the MAPA Technical Note, with a reference to one study which, ABIA claimed, showed that the Brazilian Guide is "one of the worst on the planet."

The spurious "Technical Note” and ABIA claims have been followed by an overwhelming number of statements of support for the Guide, from Brazil and worldwide. Three examples follow.

As reported in the Estado de Sao Paulo (Cambricoli, 2020), scientists from the universities of Harvard (USA) and Oxford (UK), authors of the study quoted as a "proof" that the Brazilian Guide is one of the worst on the planet, have denounced ABIA, accusing it "of dishonesty for using the results of their research in a distorted way to attack the Brazilian guide.” Marco Springmann, the Oxford author, said: "The industry article cites a classification that does not exist." Anna Herforth, the Harvard author, said: "We study dietary guidelines because they are important policy documents that help put into practice the scientific consensus on healthy diets. These documents should be based on science, not industry lobbies." She also said that their study supports the Brazilian Guide's statement that consumption of ultra-processed foods is associated with a higher risk of chronic diseases.

Thirty-three distinguished scholars from the USA, Canada, the UK, Australia, New Zealand, South Africa, Mexico, and Chile, many of whom advise the governments of their countries, wrote to the Minister of Agriculture confirming that the Technical Note has no valid foundation. It ignores the fact that the Brazilian Dietary Guidelines, 
supervised by a multi-professional team working for the Ministry of Health Food and Nutrition Coordination Unit (CGAN), were the subject of the consultations mentioned above.

The Alliance for Adequate and Healthy Food has mobilised a total of 349 civil society organisations from all over Brazil, together with 45,983 health professionals and others concerned with public health, in support of the Guide. The Alliance states in part, "The increasing number of people affected by chronic non-communicable diseases associated with the consumption of ultra-processed foods and beverages is a reality that is not only Brazilian, but is global, as shown by extensive scientific evidence...Chronic diseases are associated with the severity and lethality of Covid-19. This further reinforces the importance of integrated food care in a period of reduction in the supply of basic services and economic crisis...[and] the need for equitable, resilient and sustainable food systems...Food systems should aim first and only at the health of people and the planet."

Following these and other statements of support, the Brazilian Minister of Agriculture Tereza Cristina has rejected the "Technical Note” as inadequate (Bergamo, 2020), and confirms that nutritional issues are the responsibility of the Health Ministry. But it is certain that the ultra-processed food manufacturers will continue to do all they can to discredit and damage the Guide.

\section{The science is conclusive}

Scientific studies published between 2010 and 2020, carried out in the USA, Canada, the UK, France, Spain, Australia, Japan, Taiwan, Chile, Colombia, Argentina, Mexico, and other countries, as well as in Brazil, consistently show that higher consumption of ultra-processed food is associated with reduction of diet quality and with increased incidence of obesity and other chronic non-communicable conditions and diseases such as diabetes, hypertension, heart disease, some cancers, and earlier mortality (RicoCampà et al., 2019).

A randomised controlled trial carried out by researchers from the US National Institutes of Health (NIH) published in 2019 (Hall et al., 2019), has found that consumption of ultra-processed foods causes increased energy intake and increased body fat and total body weight. The Director of NIH, Francis Collins, stated: “A good place to start in reaching or maintaining a healthy weight is to...work to eliminate or at least reduce ultra-processed foods in your diet in favour of a balanced variety of unprocessed, nutrient-packed foods."

Since its publication, the principles and recommendations of the Guide have been adopted by the governments of Peru, Ecuador and Uruguay and Israel, and have influenced official food and nutrition policies and programmes in Canada and France (Santé Publique France, 2019). As one example, the current recommendations recently 
published by the Israeli Ministry of Health (Lev and Rosenberg, 2020) state that ultraprocessed foods increase the risk of "diabetes, cardiovascular disease, obesity, fatty liver, certain types of cancer, damage to the microbiome, an increase in the risk of mental illness, and more." They also state that ultra-processed foods are "harmful to culture, social life, and the environment.” For these and many other stated reasons they "should be consumed as infrequently as possible."

In August this year, four systematic reviews were published in leading scientific journals. These reviews evaluate all studies on a given theme already published in scientific journals worldwide. They all confirmed that substantial consumption of ultraprocessed foods increases the risk of obesity and of diabetes, high blood pressure, cardiovascular diseases, or depression, and other chronic diseases, and also shorten life expectancy (Askari et al., 2020).

Reasons why ultra-processed foods cause so many diseases include their unbalanced nutritional profile, their physical and sensory characteristics that make them caloriedense, substances generated by high temperature and pressure conditions or released by plastic packaging, and the presence of emulsifiers and other additives that may damage the gut microbiome (Lawrence and Baker, 2019).

The case for fresh and minimally processed food, made into delicious dishes and meals as still enjoyed by tens of millions of Brazilian families, and against ultra-processed food, is rock-solid.

The Golden Rule contained in the Dietary Guidelines for the Brazilian Population is indisputable. "Always prefer fresh or minimally processed foods and culinary preparations to ultra-processed foods.” So simple, as healthy eating should always be.

\section{References}

Askari M, Heshmati J, Shahinfar H, et al. 2020. Ultra-processed food and the risk of overweight and obesity: a systematic review and meta-analysis of observational studies. Int J Obes 44:2080-2091. doi: https://doi.org/10.1038/s41366-020-00650-z

Bergamo M. 2020. “Tereza Cristina rejeita nota técnica que critica Guia Alimentar”, Folha de S. Paulo, 25 September, p. B4.

Ministério da Saúde, Brasil. 2014. Guia Alimentar para a População Brasileira promovendo a alimentação saudável. Normas e manuais técnicos: Brasília.

Cambricoli F. 2020. "Entidade deturpa estudo sobre guia de comida, diz cientista”, O Estado de S. Paulo, 22 September, p. A13. 
Hall K, Ayuketah A, et al. 2019. Ultra-processed diets cause excess calorie intake and weight gain: an inpatient randomized controlled trial of ad libitum food intake. Cell Metab. 30(1):67-77.e3. doi: 10.1016/j.cmet.2019.05.008

Lawrence Mark A, Baker Phillip I. 2019. Ultra-processed food and adverse health outcomes. BMJ 365:12289. doi: 10.1136/bmj.12289

Lev B, Rosenberg E, et al. 2020. Health Behaviors: Healthful Nutrition. Jerusalem: Ministry of Health.

Rico-Campà A, Martínez-González Miguel A, Alvarez-Alvarez I, et al. 2019.

Association between consumption of ultra-processed foods and all-cause mortality: SUN prospective cohort study. BMJ 365:11949. doi: 10.1136/bmj.11949

Santé Publique France. 2019. Santé publique France présente les nouvelles recommandations sur l'alimentation, l'activité physique et la sédentarité. Retrieved from: https://www.santepubliquefrance.fr/presse/2019/sante-publique-france-presente-lesnouvelles-recommandations-sur-l-alimentation-l-activite-physique-et-la-sedentarite 